\title{
Single-Transverse-Spin-Asymmetry studies with a fixed-target experiment using the LHC beams (AFTER@LHC)
}

\section{Jean-Philippe Lansberg;, on behalf of the AFTER@LHC study group ${ }^{\dagger}$}

We discuss the potential of AFTER@LHC to measure single-transverse-spin asymmetries in open-charm and bottomonium production. With a HERMES-like hydrogen polarised target, such measurements over a year can reach precisions close to the per cent level. This is particularly remarkable since these analyses can probably not be carried out anywhere else.

XXIV International Workshop on Deep-Inelastic Scattering and Related Subjects

11-15 April, 2016

DESY Hamburg, Germany

*Jean-Philippe.Lansberg@in2p3.fr

${ }^{\dagger}$ : M. Anselmino, R. Arnaldi, D. Boer, S.J. Brodsky, V. Chambert, C. Da Silva, J.P. Didelez, M.G. Echevarria, E.G. Ferreiro, F. Fleuret, Y. Gao, B. Genolini, C. Hadjidakis, I. Hrivnacova, D. Kikola, A. Klein, A. Kurepin, O. Kusina, R. Laha, F. Lyonnet, L. Massacrier, C. Lorcé, A. Nass, C. Quintans, P. Robbe, I. Schienbein, E. Scomparin, J. Seixas, H.S. Shao, A. Signori, E. Steffens, N. Topilskaya, B. Trzeciak, U.I. Uggerhøj, R. Ulrich, Z. Yang. 


\section{Introduction}

Continuous efforts have been made in the last decades to advance our knowledge of the internal structure of the nucleon and, in particular, that of the constituent dynamics, the quarks and the gluons. However, it remains largely unknown with a limited understanding of the proton and neutron spin structure, namely how they bind into a spin- $\frac{1}{2}$ object. There are two types of quark and gluon contributions to the nucleon spin: their spin and their Orbital Angular Momentum (OAM). For a $+\frac{1}{2}$ helicity nucleon, one has $\frac{1}{2}=\frac{1}{2} \Delta \Sigma+\Delta G+\mathscr{L}_{g}+\mathscr{L}_{q}$ where $\frac{1}{2} \Delta \Sigma$ refers to the combined spin contribution of quarks and antiquarks, $\Delta G$ the gluon spin, and $\mathscr{L}_{q, g}$ the quark and gluon OAM contributions (see e.g. $[1,2]$ ). This equation applies whatever the energy scale we look at the nucleons and this strongly motivates the scale-evolution study of the individual contributions.

The spin crisis of the 80 's has evolved into a puzzle, i.e. to determine how large the quark and gluon contributions to the nucleon spin are, to disentangle them and eventually to explain them from first principles in QCD. Recent experimental analyses pointed at $\Delta \Sigma$ as low as 0.25 [3] and that $\Delta G$ could $^{1}$ reach 0.2 . There is still ample room -rather, need- for $\mathscr{L}_{q}$ and $\mathscr{L}_{g}$, which have not yet been measured. This highlights how important studies of the transverse motion of quarks and gluons inside the proton are. Indirect ${ }^{2}$ information on the orbital motion of the partons bound inside hadrons can be accessed via Single (Transverse) Spin Asymmetries (S(T)SA) in different hardscattering processes, in particular with a transversely polarised hadron (see [6,7] for recent reviews) to probe the Sivers effect $[8,9]^{3}$. This effect is naturally connected $[10,11]$ to the transverse motion of the partons inside the polarised nucleons (see also [12]).

As of today, the Sivers effect can be approached via two dual formalisms [13]. One extends the collinear parton model of Bjorken with quark-gluon or gluon-gluon correlation functions [14-16]. It is called the Collinear Twist-3 (CT3) formalism. Another, referred to as the Transverse-Momentum Dependent (TMD) factorisation (see e.g. [17-19]), uses a complete tridimensional mapping of the parton momentum. It can also deal with all the possible spin-spin and spin-orbit correlations between the hadron and the partons. Both approaches encode the rescatterings ${ }^{4}$ of the quarks and gluons with the hadron remnants [20-22] which are believed to generate the STSAs, also referred to $A_{N}$. Both formalisms have their preferred range of applicability. As for the CT3 observables, the AFTER@LHC physics case adds up heavy-flavour and bottomonium production, whose STSAs are essentially unknown, to the usual list of $A_{N}$ studies which includes single hadron [23], Drell Yan (DY) pair [24-26] or isolated photon [27] production. For TMD observables, one usually looks at processes immune to final-state radiations which allows one to control the transverse momentum of the initial partons by measuring momentum imbalances. In the case of AFTER@LHC, the possible measurements go well beyond DY produc-

\footnotetext{
${ }^{1}$ The latter has only been probed for $x>0.05[4,5]$

${ }^{2}$ To measure the parton OAM, observables which are sensitive to the parton position and momentum are in principle required. A well known example of such objects are Generalised Parton Distributions (GPDs) accessible in exclusive processes.

${ }^{3}$ It is connected to left-right asymmetries in the parton distributions with respect to the plane formed by the proton momentum and spin directions

${ }^{4}$ They are accounted by gauge links in the definition of the TMD PDFs and explicitly considered in the hardscattering coefficient for CT3.
} 
tion and include pseudo-scalar quarkonium, quarkonium-pair and other associated production of colourless particles.

It is noteworthy that the TMD factorisation approach also allows one to investigate the structure of hadrons in a tridimensional momentum space (see [19] and references therein) in a rigorous and systematic way and it is not restricted to the study of STSAs. It provides theoretical tools to directly study not only the transverse-momentum distributions of the partons but also their polarisation in both polarised and unpolarised nucleons. Such studies are in particular related to azimuthal asymmetries in the final state. At AFTER@LHC [28], one can study them, for instance, via pseudo-scalar quarkonium production [29,30], associated quarkonium production $[31,32]$ and, of course, DY pair production [33-35]. We guide the reader to [36,37] for more details.

With its high luminosity, a highly polarised target and an access towards the large momentum fraction $x^{\uparrow}$ in the target, AFTER @LHC is probably the best set-up $[28,38]$ to carry out an inclusive set of $A_{N}$ and azimuthal asymmetry measurements both to improve existing analyses and to perform studies which are simply impossible elsewhere. Let us recall that nearly nothing is known from the experimental side about the gluon Sivers effect (see e.g. [39] for a recent review). The polarisation of not only hydrogen but also deuterium and helium targets allows for an even more ambitious spin program bearing on the neutron and spin 1 bound states [40].

\section{Experimental implementation}

Two approaches for a fixed-target experiment at the LHC with a polarised target can be considered: one with a bent-crystal extracted beam on a conventional polarised target, the other with an internal polarised gas target. Both have the virtue of being parasitic for they do not affect the LHC performances. In the following, we will focus on the latter option ${ }^{5}$ which can directly be combined with existing detectors such as LHCb or ALICE. In particular, we will rely on the expected performances of a target system like the one of the HERMES experiment at DESY-HERA [42], as proposed in [43].

\begin{tabular}{cccccc} 
Beam & Target & $A$ & $\begin{array}{c}\text { Areal density }(\theta) \\
\left(\mathrm{cm}^{-2}\right)\end{array}$ & $\begin{array}{c}\mathscr{L} \\
\left(\mu \mathrm{b}^{-1} \cdot \mathrm{s}^{-1}\right)\end{array}$ & $\begin{array}{c}\int \mathscr{L} \\
\left(\mathrm{fb}^{-1} \cdot \mathrm{y}^{-1}\right)\end{array}$ \\
\hline$p$ & $\mathrm{H}$ & 1 & $2.5 \times 10^{14}$ & 900 & 9 \\
$p$ & $\mathrm{D}$ & 2 & $3.2 \times 10^{14}$ & 1200 & 12
\end{tabular}

Table 1: Expected luminosities with an internal gas-target inspired by the HERMES experiment.

In such a case, the luminosity is given by the product of the particle current $I$ and the areal density of the polarised storage cell target, $\theta$. The density results from many parameters, the flux of the polarised source injected into the target cell and the cell geometry are the most relevant ones [44]. For a LHC compatible set-up [44] and taking the HERMES-target-source flux, one can expect a density of $2.5 \times 10^{14} \mathrm{~cm}^{-2}$ for a $1 \mathrm{~m}$ long cell for $\mathrm{H}$ at $300 \mathrm{~K}$ and, for $\mathrm{D}, 3.2 \times$

\footnotetext{
${ }^{5}$ Such an internal gas-target option is in fact currently used by the LHCb collaboration but as a luminosity monitor [41] (SMOG) initially designed to study the transverse size of the beam. Despite its limited pressure (about $10^{-7} \mathrm{mbar}$ ), it acts as an ideal demonstrator of such a solution over extended periods of time, without any interferences on the other LHC experiments. LHCb performed pilot runs with $p$ and $\mathrm{Pb}$ beams on a $\mathrm{Ne}$ gas target in 2012 and 2013. These pilot runs were followed by longer runs in 2015: $p$ on Ne (12 hours), He (8 hours) and Ar ( 3 days) as well as $\mathrm{Pb}$ on $\mathrm{Ar}$ (1 week) and $p$ on $\mathrm{Ar}$ (a few hours). As for now, the current gas pressure is limited by the pumping system.
} 
$10^{14} \mathrm{~cm}^{-2}$. This is well below the densities which might affect the proton beam life time. The resulting luminosities with $I=3.14 \times 10^{18} p^{+} \mathrm{s}^{-1}$ for the $p^{+}$beam case are shown in Table 1 . The limit of such a solution is essentially set by the number of minimum bias collisions by fill, that is the number of proton "consumed" by the target as compared to the total number stored in the LHC.

In terms of the polarisation, by reusing a target like that of HERMES, the effective polarisation of $\mathrm{H}$ or D [43] can be as high as $0.8 .{ }^{3} \mathrm{He}$ can also be used. In the following, we will take an effective polariation of $P=0.6$ as a working hypothesis which, from the number above, is definitely a conservative assumption.

\section{Selected figures-of-merit for STSAs}

In this section, we quickly discuss figures-of-merit for open charm and bottomonium production. Those for Drell-Yan and $J / \psi$ can be found in [38]. They both show that $A_{N}$ can be measured close to the per cent level in regions where the expected effects can be as large as ten or even twenty per cent.

\subsection{STSAs in open (anti)-charm production}

Some years ago, it was argued [45] that the study of STSAs in open heavy-flavour production in the RHIC energy domain gives a direct access to the tri-gluon correlations functions appearing in the CT3 approach. Such correlations can be related to the gluon Sivers functions under some assumptions. If STSAs for charm quarks and anti-quarks can separately be measured, this will also be a unique probe of $C$-parity odd twist-3 tri-gluon correlators [46,47].
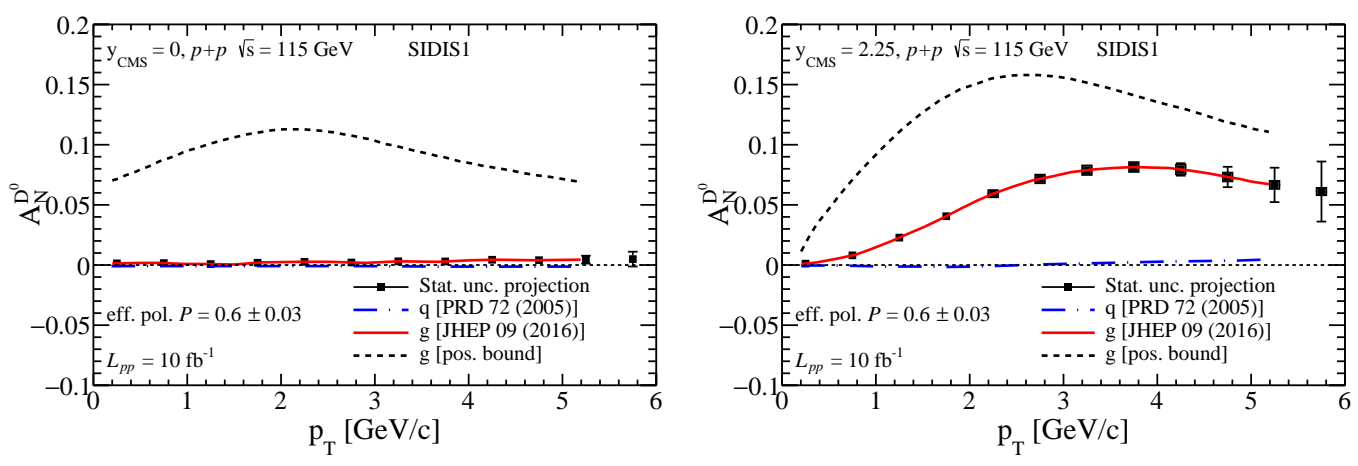

Figure 1: Projected $P_{T}$ dependence of $A_{N}$ for $D^{0}$ at $y_{\text {c.m.s. }}=0\left(y_{\text {c.m.s. }}=2.25\right)$ on the left (middle).

Fig. 1 (left \& middle) shows the projected statistical precision ${ }^{6}$ for $D^{0}$ meson STSAs at AFTER@LHC. It definitely lies at the per cent level. The expected magnitude is from [49,50]. As for now, such measurements are not planned elsewhere, certainly not in the large $x^{\uparrow}$ region where the STSAs are expected to be the largest.

\subsection{STSAs in bottomonium production}

Bottomonia can also provide much information on the Sivers effects in the gluon sector, especially via STSAs analyses of the $\Upsilon(1 S), \Upsilon(2 S), \Upsilon(3 S)$ which contain different amounts of $P$-wave

\footnotetext{
${ }^{6}$ In view of very low level of background in various $D^{0} \mathrm{LHCb}$ analyses, we neglected it here. We have also assumed LHCb-like acceptance and performances, see [48].
} 
feed-downs. We believe that STSAs of $\Upsilon(2 S)$ and $\Upsilon(3 S)$ would not be accessible anywhere else than at AFTER@LHC. Since only one particle is observed, these STSAs are preferably treated in the CT3 approach that was, for instance, applied to the $\eta_{c}$ case [51] which could also be measured at AFTER@LHC. As for now, quantitive predictions for these STSAs are lacking even though the qualitative conclusion of [52] should apply and the observation of a non-zero STSA should already provide us with new information both on the quarkonium-production mechanisms [53-55] and on the gluon Sivers effect [39]. For the figure-of-merit displayed on Fig. 2, we have thus set the central value of the points to $A_{N}=0$. It clearly shows that, even for the least produced $\Upsilon(3 S)$, the statistical precision is better than 5\% taking into account a full background simulation as discussed in [56].

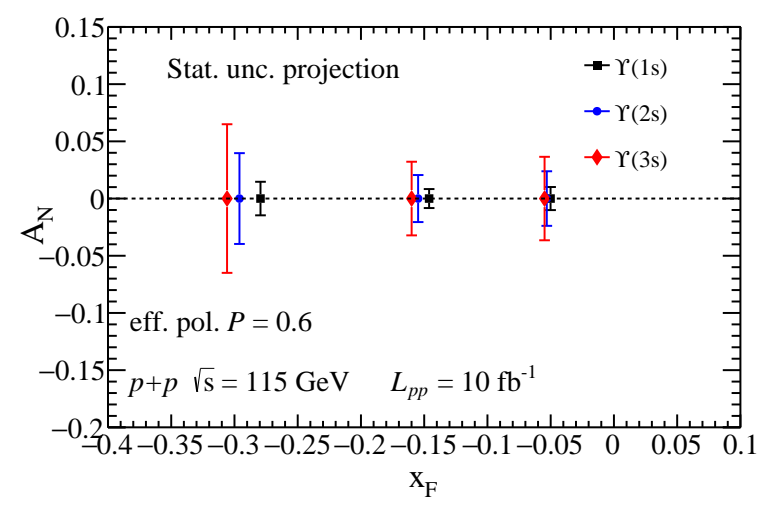

Figure 2: Projected $x_{F}$ dependence of the statistical uncertainty on $A_{N}$ for $\Upsilon(1 S), \Upsilon(2 S), \Upsilon(3 S)$ corresponding to 3 bins in rapidity $([2: 3],[3: 4],[4: 5])$.

\section{Conclusion}

The combinaison of the TeV LHC proton beam, of a polarised internal gas-target system inspired by that of the HERMES experiment and a detector like LHCb (or ALICE) opens the way to a number of outstanding STSA measurements at the per cent level, most of which cannot be carried out at other facilities.

As concluding remarks, we would like to emphasise two important facts: (i) at AFTER@LHC, these STSAs would naturally be measured at large $x^{\uparrow}$ where the (quark and gluon) Sivers effect is expected to be the largest and (ii) such measurements have to be measured in $p p$ collisions as mandatory complementary pieces of information to similar studies in lepton-induced reactions to perform quantitative tests of the generalised universality of the TMD-related observables, deeply connected to QCD.

\section{References}

[1] E. Leader, C. Lorce, Phys. Rept. 541, 163 (2014).

[2] M. Wakamatsu, Int. J. Mod. Phys. A29, 1430012 (2014).

[3] D. de Florian, R. Sassot, M. Stratmann, W. Vogelsang, Phys. Rev. Lett. 101, 072001 (2008).

[4] L. Adamczyk, et al., Phys. Rev. Lett. 115, 092002 (2015).

[5] D. de Florian, R. Sassot, M. Stratmann, W. Vogelsang, Phys. Rev. Lett. 113, 012001 (2014).

[6] U. D’Alesio, F. Murgia, Prog. Part. Nucl. Phys. 61, 394 (2008).

[7] V. Barone, F. Bradamante, A. Martin, Prog. Part. Nucl. Phys. 65, 267 (2010).

[8] D. W. Sivers, Phys. Rev. D41, 83 (1990). 
[9] D. W. Sivers, Phys. Rev. D43, 261 (1991).

[10] M. Burkardt, Phys. Rev. D66, 114005 (2002).

[11] M. Burkardt, Nucl. Phys. A735, 185 (2004).

[12] K.-F. Liu, C. Lorce, Eur. Phys. J. A52, 160 (2016).

[13] Y. Koike, W. Vogelsang, F. Yuan, Phys. Lett. B659, 878 (2008).

[14] A. V. Efremov, O. V. Teryaev, Sov. J. Nucl. Phys. 36, 140 (1982). [Yad. Fiz.36,242(1982)].

[15] A. V. Efremov, O. V. Teryaev, Phys. Lett. B150, 383 (1985).

[16] J.-w. Qiu, G. F. Sterman, Phys. Rev. Lett. 67, 2264 (1991).

[17] J. Collins, Foundations of perturbative QCD (Cambridge University Press, 2013).

[18] M. G. Echevarria, A. Idilbi, I. Scimemi, JHEP 07, 002 (2012).

[19] R. Angeles-Martinez, et al., Acta Phys. Polon. B46, 2501 (2015).

[20] S. J. Brodsky, D. S. Hwang, I. Schmidt, Nucl. Phys. B642, 344 (2002).

[21] J. C. Collins, Phys. Lett. B536, 43 (2002).

[22] S. J. Brodsky, D. S. Hwang, I. Schmidt, Phys. Lett. B530, 99 (2002).

[23] J.-w. Qiu, G. F. Sterman, Phys. Rev. D59, 014004 (1999).

[24] N. Hammon, O. Teryaev, A. Schafer, Phys. Lett. B390, 409 (1997).

[25] D. Boer, P. J. Mulders, O. V. Teryaev, Phys. Rev. D57, 3057 (1998).

[26] D. Boer, P. J. Mulders, Nucl. Phys. B569, 505 (2000).

[27] J.-w. Qiu, G. F. Sterman, Nucl. Phys. B378, 52 (1992).

[28] S. J. Brodsky, F. Fleuret, C. Hadjidakis, J. P. Lansberg, Phys. Rept. 522, 239 (2013).

[29] D. Boer, C. Pisano, Phys. Rev. D86, 094007 (2012).

[30] A. Signori, Few Body Syst. 57, 651 (2016).

[31] W. J. den Dunnen, J. P. Lansberg, C. Pisano, M. Schlegel, Phys. Rev. Lett. 112, 212001 (2014).

[32] J.-P. Lansberg, H.-S. Shao, Nucl. Phys. B900, 273 (2015).

[33] T. Liu, B.-Q. Ma, Eur. Phys. J. C72, 2037 (2012).

[34] M. Anselmino, U. D’Alesio, S. Melis, Adv. High Energy Phys. 2015, 475040 (2015).

[35] K. Kanazawa, Y. Koike, A. Metz, D. Pitonyak, Adv. High Energy Phys. 2015, 257934 (2015).

[36] J. P. Lansberg, et al., EPJ Web Conf. 85, 02038 (2015).

[37] L. Massacrier, et al., Int. J. Mod. Phys. Conf. Ser. 40, 1660107 (2016).

[38] J.-P. Lansberg, et al., PoS PSTP2015, 042 (2016).

[39] D. Boer, C. Lorce, C. Pisano, J. Zhou, Adv. High Energy Phys. 2015, 371396 (2015).

[40] D. Boer, et al. (2016).

[41] M. Ferro-Luzzi, Nucl. Instrum. Meth. A553, 388 (2005).

[42] A. Airapetian, et al., Nucl. Instrum. Meth. A540, 68 (2005).

[43] C. Barschel, P. Lenisa, A. Nass, E. Steffens, Adv. High Energy Phys. 2015, 463141 (2015).

[44] E. Steffens, PoS PSTP2015, 019 (2015).

[45] Z.-B. Kang, J.-W. Qiu, W. Vogelsang, F. Yuan, Phys. Rev. D78, 114013 (2008).

[46] X.-D. Ji, Phys. Lett. B289, 137 (1992).

[47] H. Beppu, Y. Koike, K. Tanaka, S. Yoshida, Phys. Rev. D82, 054005 (2010).

[48] D. Kikola, Adv. High Energy Phys. 2015, 783134 (2015).

[49] M. Anselmino, M. Boglione, U. D’Alesio, E. Leader, F. Murgia, Phys. Rev. D70, 074025 (2004).

[50] U. D’Alesio, F. Murgia, C. Pisano, JHEP 09, 119 (2015).

[51] A. Schafer, J. Zhou, Phys. Rev. D88, 014008 (2013).

[52] F. Yuan, Phys. Rev. D78, 014024 (2008).

[53] A. Andronic, et al., Eur. Phys. J. C76, 107 (2016).

[54] N. Brambilla, et al., Eur. Phys. J. C71, 1534 (2011).

[55] J. P. Lansberg, Int. J. Mod. Phys. A21, 3857 (2006).

[56] L. Massacrier, et al., Adv. High Energy Phys. 2015, 986348 (2015). 